\title{
Перенос заряда в тонких слоях стеклообразной гибридной системы $\mathrm{Ge}_{28.5} \mathrm{~Pb}_{14.5} \mathrm{Fe}_{0.5} \mathrm{~S}_{56.5}$
}

\author{
(C) Р.А. Кастро-Арата ${ }^{1}$, Г.И. Грабко ${ }^{1,2}$, А.А. Кононов ${ }^{1}$, Н.И. Анисимова ${ }^{1}$, М. Крбал ${ }^{3}$, А.В. Колобов ${ }^{1,4}$ \\ ${ }^{1}$ Российский государственный педагогический университет им. А.И. Герцена, \\ 191186 Санкт-Петербург, Россия \\ 2 Забайкальский государственный университет, \\ 672039 Чита, Россия \\ ${ }^{3}$ Университет Пардубице, \\ 53002 Пардубице, Чехия \\ ${ }^{4}$ Национальный институт передовых промышленных наук и технологий, \\ 305-8565 Цукуба, Япония \\ E-mail: recastro@mail.ru
}

Поступила в Редакцию 18 января 2021 г.

В окончательной редакции 25 января 2021 г.

Принята к публикации 25 января 2021 г.

Представлены результаты исследования процессов переноса заряда в тонких слоях халькогенидной гибридной системы $\mathrm{Ge}_{28.5} \mathrm{~Pb}_{14.5} \mathrm{Fe}_{0.5556 .5}$. Обнаружены степенная зависимость удельной проводимости от частоты и уменьшение значения показателя степени s c увеличением температуры. Перенос заряда является термически активированным процессом с энергией активации $E_{a}=(0.64 \pm 0.02)$ эВ. Полученные результаты объясняются в рамках модели проводимости в неупорядоченных системах $\mathrm{CBH}$ (correlated barrier hopping). Рассчитанная доля неподеленных электронных пар указывает на то, что при введении железа материал остается в стеклообразующей области.

Ключевые слова:: прыжковый механизм переноса, тонкие слои, стеклообразная гибридная система, неподеленные электронные пары.

DOI: $10.21883 /$ FTP.2021.05.50836.9578

\section{1. Введение}

Халькогенидные стеклообразные полупроводники (ХСП) сложного состава привлекают внимание исследователей в связи с их использованием в многочисленных приспособлениях микро- и оптоэлектроники. Например, в настоящее время ХСП используются при изготовлении тепловых систем отображения [1], волокон и плоских волноводов, прозрачных в инфракрасном диапазоне [2], в оптических сенсорах [3] и нелинейной оптике [4].

Классические ХСП удовлетворяют правилу Мотта $8-N[5]$ (где $N$ - число валентных электронов), в соответствии с которым атомы селена являются двухкоординированными $(8-6=2)$, притом что два $p$-электрона образуют так называемую неподеленную электронную пару (НЭП), не участвующую в образовании ковалентных связей. Особая роль неподеленных пар была впервые отмечена Кастнером, предложившим для описания ХСП термин „полупроводники с неподеленными парами“ (lone-pair semiconductors [6]). При этом электроны неподеленных пар формируют верх валентной зоны и, как следствие, играют весьма важную роль в формировании многих свойств, в частности формируют способность ХСП к фотостимулированному изменению структуры [7]. На решающую роль неподеленных пар в явлениях переключения и памяти указывается в рабо$\operatorname{Tax}[8,9]$.
C другой стороны, в последние годы наблюдается огромный интерес к двумерным халькогенидам, таким как топологические изоляторы типа $\mathrm{Sb}_{2} \mathrm{Te}_{3}[10]$ и дихалькогениды переходных металлов (ПМ) [11]. Последние иногда называют „суперматериалом, который может победить графен“ [12]. Характер связей в этих материалах отличается от ХСП. Так, в кристаллических дихалькогенидах ПМ все неподеленные пары халькогена задействованы на образование химических связей, свободных неподеленных пар в материале нет.

В этой связи представляется чрезвычайно интересным исследование структуры и свойств гибридных аморфных полупроводников, т.е. классических ХСП, модифицированных ПМ, что позволит управлять концентрацией неподеленных пар в материале и таким образом контролировать его стеклообразующую способность (и другие свойства). Цель данной работы заключалась в выявлении особенностей процессов переноса заряда в тонких слоях стеклообразной системы $\mathrm{Ge}_{28.5} \mathrm{~Pb}_{14.5} \mathrm{Fe}_{0.5} \mathrm{~S}_{56.5}$ методом низкочастотной диэлектрической спектроскопии.

\section{2. Теория}

Известно, что в неупорядоченных средах при приложении слабого переменного электрического поля для области низких частот $\left(\omega<10^{7}\right.$ Гц $)$ можно выделить два основных механизма переноса заряда: 1) перенос носителями, возбужденными в локализованные состояния 
вблизи краев валентной зоны или зоны проводимости; 2) прыжковый перенос носителями с энергиями вблизи уровня Ферми [13]. Частотная зависимость вещественной части удельной проводимости описывается степенным законом:

$$
\sigma^{\prime}(\omega)=A \omega^{s}
$$

где $\omega-$ угловая частота $(\omega=2 \pi f), A-$ независимая от частоты постоянная, $s$ - показатель степени.

Согласно модели СВН (correlated barrier hopping model) [14], электроны совершают прыжки между заряженными состояниями, при этом выражение для проводимости на переменном токе для конкретной фиксированной температуры имеет вид [15]

$$
\sigma^{\prime}(\omega)=\frac{\pi^{3} N^{2} \varepsilon \varepsilon_{0} \omega R_{\omega}^{6}}{24}
$$

где $N$ - плотность состояний, между которыми совершают прыжки носители заряда. Связь между длиной прыжка $R_{\omega}$ и высотой потенциального барьера выражается соотношением

$$
R_{\omega}=\frac{e^{2}}{\pi \varepsilon \varepsilon_{0}}\left[W_{M}-k T \ln \left(\frac{1}{\omega \tau_{0}}\right)\right]^{-1},
$$

где $\tau_{0}-$ характеристическое время релаксации, т.е. величина, обратная фононной частоте $v_{\mathrm{ph}}$. С другой стороны, показатель степени $s$ связан с высотой барьера $W_{M}$ через выражение

$$
s=1-\frac{6 k T}{\left[W_{M}-k T\left(1 / \omega \tau_{0}\right)\right]} .
$$

Уравнение (4) можно упростить в первом приближении [16]:

$$
s=1-\frac{6 k T}{W_{M}} .
$$

\section{3. Методика эксперимента}

Измерения диэлектрических спектров проводили на тонкопленочных образцах халькогенидной системы $\mathrm{Ge}_{28.5} \mathrm{~Pb}_{14.5} \mathrm{Fe}_{0.5} \mathrm{~S}_{56.5}$. Тонкие пленки были получены методом „ударного“ напыления в вакууме, при котором частицы размером $\sim 2$ мкм малыми порциями непрерывно попадают в разогретый испаритель. Испарение проводилось при давлении остаточных газов $10^{-5}$ мм рт. ст. Напыление осуществлялось на стеклянные подложки, удаленные на расстояние 15-30 см от испарителя. Данная технология позволяет получать тонкопленочные образцы однородного состава по толщине пленки [17]. Образцы имели сандвич-конфигурацию $\mathrm{Au}-\mathrm{XC}$ П-Al c площадью контактов $14.0 \mathrm{Mм}^{2}$. Толщина слоев, определяемая с помощью спектрального эллипсометра ЭЛЬФ, составляла $\sim 2.0$ мкм. Степень аморфности образцов контролировали с применением рентгеновского дифрактометра ДРОН-7. Измерения проводили на монохроматическом рентгеновском излучении $\mathrm{Cu} K_{\alpha 1}$ с длиной

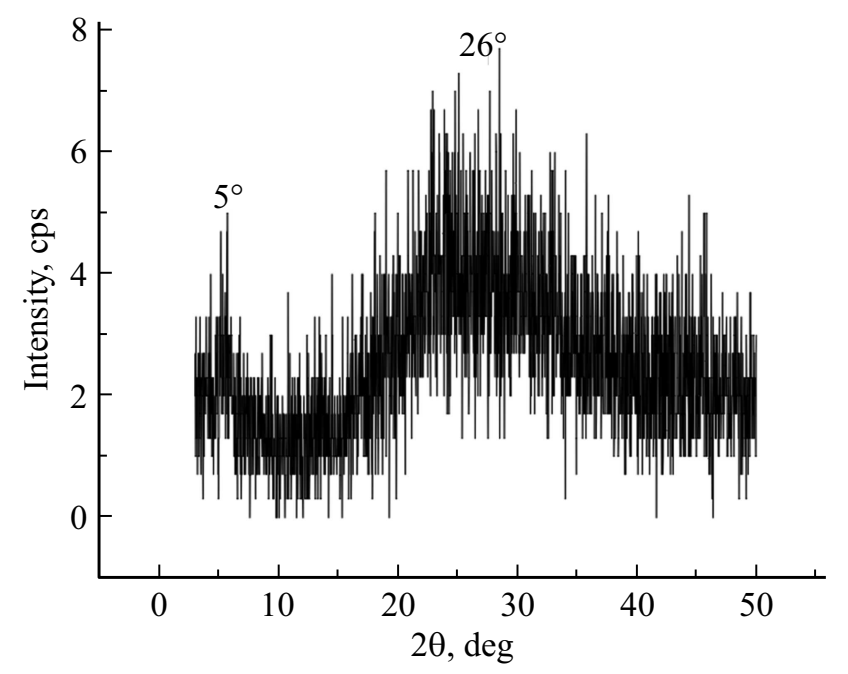

Рис. 1. Рентгенограмма образца \#3 полупроводниковой пленки $\mathrm{Ge}_{28.5} \mathrm{~Pb}_{14.5} \mathrm{Fe}_{0.5} \mathrm{~S}_{56.5}$ с указанием углов рассеяния $2 \theta$.

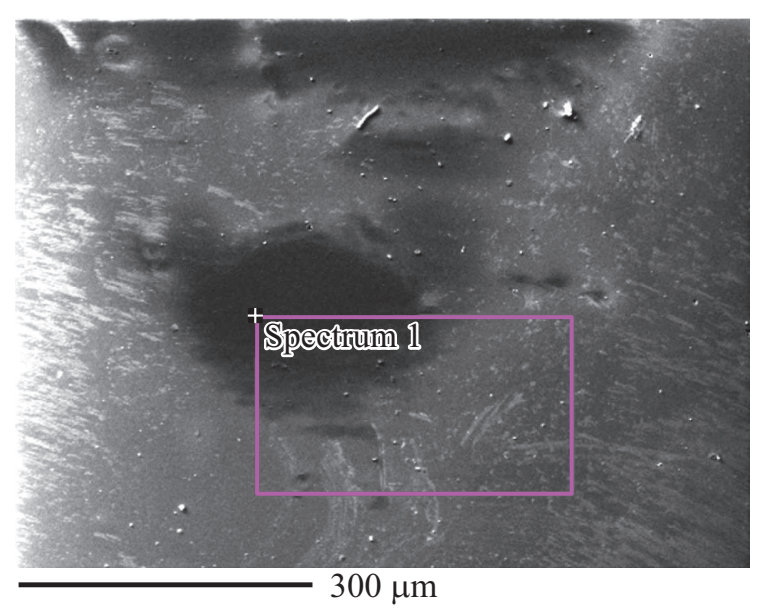

Рис. 2. Скан поверхности образца гибридной системы $\mathrm{Ge}_{28.5} \mathrm{~Pb}_{14.5} \mathrm{Fe}_{0.5} \mathrm{~S}_{56.5}$.

волны $\lambda=1.5406 \AA$. Полученные рентгенограммы, измеренные при больших углах рассеяния рентгеновских лучей $2 \theta$ в диапазоне от 10 до $80^{\circ}$, указывают на аморфный характер исследуемых пленок (рис. 1).

Исследование элементного состава образцов проводилось с использованием сканирующего электронного микроскопа (SEM) Carl Zeiss EVO 40. Для определения элементного состава были выбраны точки на сканах, для которых получены спектры, отражающие соотношение атомарных концентраций элементов в образцах (рис. 2).

В табл. 1 приводятся данные по содержанию химических элементов в исследуемых образцах. Полученное соотношение элементов в стекле позволяет заключить, что исследуемая система соответствует составу $\mathrm{Ge}_{28.5} \mathrm{~Pb}_{14.5} \mathrm{Fe}_{0.5} \mathrm{~S}_{56.5}$.

Получение частотных зависимостей проводимости исследуемых слоев при разных температурах осуществлялось на спектрометре „Concept-81“ (Novocontrol 
Таблица 1. Элементный состав образцов системы $\left(\mathrm{Ge}_{28.5} \mathrm{~Pb}_{15.0} \mathrm{~S}_{56.5}\right)_{100-x} \mathrm{Fe}_{x}$

\begin{tabular}{c|c}
\hline Элемент & Атомная доля, \% \\
\hline $\mathrm{S}$ & 56.48 \\
$\mathrm{Ge}$ & 28.52 \\
$\mathrm{~Pb}$ & 14.45 \\
$\mathrm{Fe}$ & 0.55
\end{tabular}

Technologies $\mathrm{GmbH}$ ), предназначенном для исследования электрофизических свойств широкого класса материалов. Измерения проводили в диапазонах частот $f=10^{-1}-10^{6}$ Гц и температур $T=273-403 \mathrm{~K}$. Амплитуда приложенного к образцам напряжения $U=10^{-1} \mathrm{~B}$. В качестве экспериментальных данных выступали значения мнимой $\left(Z^{\prime \prime}\right)$ и действительной $\left(Z^{\prime}\right)$ частей импеданса ячейки с измеряемым образцом:

$$
Z^{*}(\omega)=Z^{\prime}+i Z^{\prime \prime}=R+\frac{1}{i \omega C}=\frac{U_{0}}{I^{*}(\omega)} .
$$

Спектры комплексной проводимости рассчитывали из спектров импеданса по формуле

$$
\sigma^{*}=\sigma^{\prime}-i \sigma^{\prime \prime}=\frac{-i}{\omega Z^{*}(\omega)} \frac{S}{d}
$$

Относительная погрешность эксперимента не превышала $5 \%$.

\section{4. Результаты и обсуждение}

На рис. 3 представлена частотная зависимость действительной части удельной проводимости $\sigma^{\prime}$ слоев

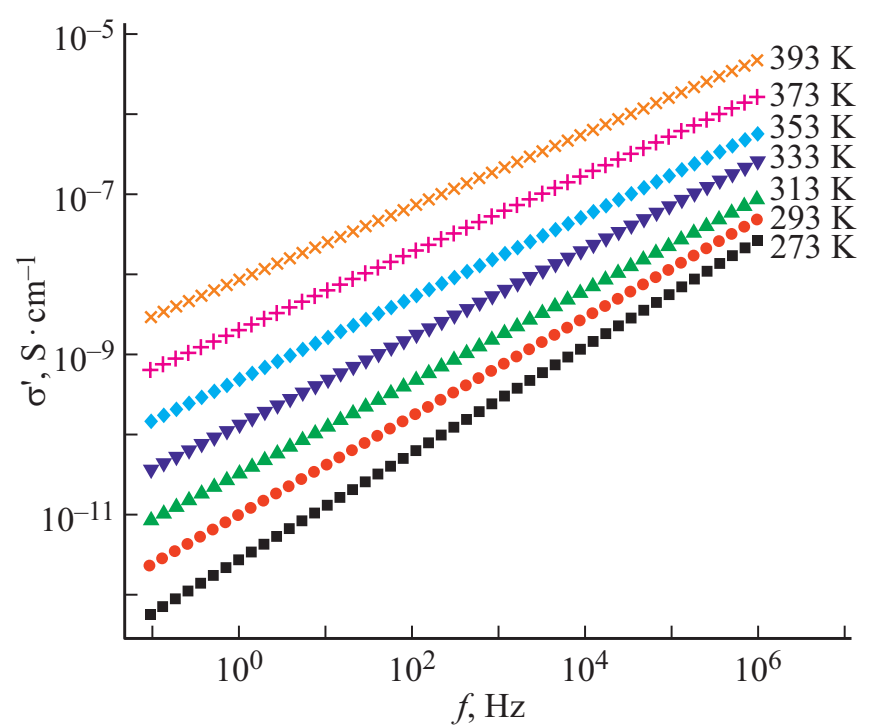

Рис. 3. Частотная зависимость удельной проводимости $\sigma^{\prime}$ при разных температурах.

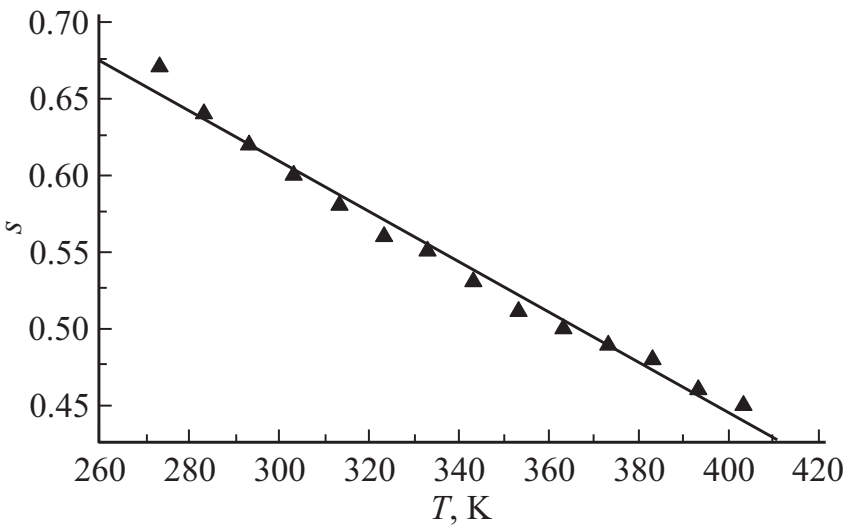

Рис. 4. Температурная зависимость показателя степени $s$.

$\mathrm{Ge}_{28.5} \mathrm{~Pb}_{14.5} \mathrm{Fe}_{0.5} \mathrm{~S}_{56.5}$ при разных температурах, из которой следует увеличение $\sigma^{\prime}$ с ростом частоты. Данная зависимость является типичной для большинства аморфных полупроводников, для которых проводимость на переменном токе меняется с частотой по закону (1).

В случае исследуемой системы показатель степени принимает значения $s=0.45-0.67$ (рис. 4). Температурная зависимость $s$ часто используется для исследования особенностей процессов переноса заряда в различных системах. Обнаруженная степенная зависимость проводимости от частоты (1) и уменьшение значения параметра $s$ с ростом температуры позволяют предположить существование прыжкового механизма проводимости.

На основе полученных экспериментальных данных, пользуясь уравнениями (2)-(5), можно оценить значение микропараметров системы $N, R_{\omega}$ и $W_{M}$. Результаты проведенных расчетов представлены в табл. 2 .

Перенос заряда в исследуемой системе является термически активированным процессом, температурная зависимость удельной проводимости для разных частот подчиняется закону Аррениуса (рис. 5)

$$
\sigma^{\prime}=\sigma_{0} \exp \left[-\frac{E_{a}}{k T}\right]
$$

с энергией активации, равной $E_{a}=(0.64 \pm 0.02)$ эВ.

Модель СВН предполагает, что перенос заряда осуществляется посредством прыжков электронов через потенциальный барьер между двумя локализованными состояниями (центрами равновесия). При этом высота барьера между двумя центрами определяется кулоновским взаимодействием между соседними дефектными

Таблица 2. Параметры переноса заряда в тонких слоях стеклообразной системы $\mathrm{Ge}_{28.5} \mathrm{~Pb}_{13.0} \mathrm{Fe}_{2.0} \mathrm{~S}_{56.5}$

\begin{tabular}{c|c|c|c|c}
\hline$T, \mathrm{~K}$ & $s$ & $N, \mathrm{M}^{-3}$ & $R_{\omega}, \AA$ & $W_{M}$, эB \\
\hline 273 & 0.67 & $2.73 \cdot 10^{26}$ & 0.22 & 0.85 \\
293 & 0.62 & $6.21 \cdot 10^{28}$ & 1.33 & 0.77 \\
313 & 0.58 & $1.05 \cdot 10^{31}$ & 2.23 & 0.72
\end{tabular}

Физика и техника полупроводников, 2021, том 55, вып. 5 


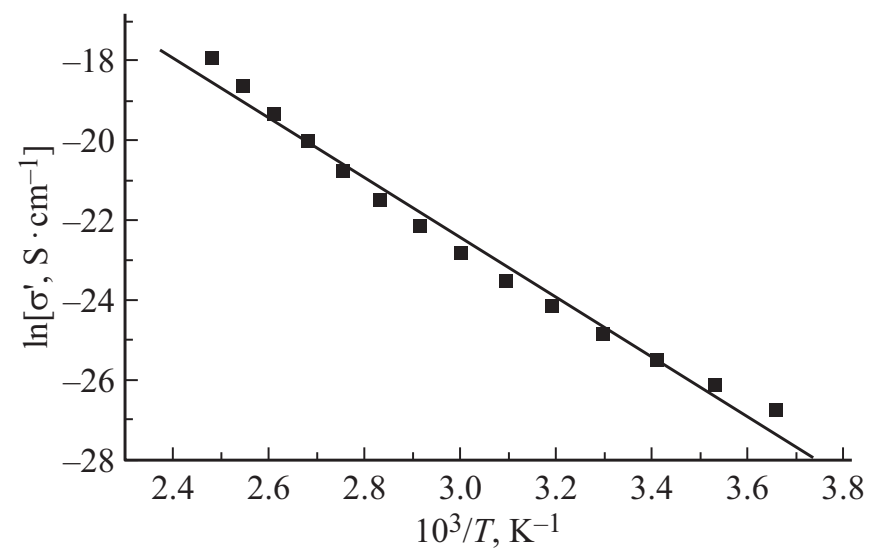

Рис. 5. Температурная зависимость удельной проводимости $\sigma^{\prime}$ на частоте $f=10^{-1}$ Гц.

состояниями, в роли которых могут выступать заряженные дефекты типа $D^{+}$и $D^{-}$, образующие диполь. В случае стеклообразной системы $\mathrm{Ge}_{28.5} \mathrm{~Pb}_{15} \mathrm{~S}_{56.5}$ обмен электронами может осуществляться, например, между атомами германия, пребывающими в двух- и четырехвалентном состояниях соответственно [18].

Авторами работы [18] показано, что введение железа в стекла сопровождается сменой типа проводимости. Проводимость чистого стекла носит дырочный характер, в то время как проводимость легированного стекла - электронный. В стеклообразных сплавах $\mathrm{Ge}_{28.5} \mathrm{~Pb}_{15-x} \mathrm{Fe}_{x} \mathrm{~S}_{56.5}$ и $\mathrm{Ge}_{27} \mathrm{~Pb}_{17-x} \mathrm{Fe}_{x} \mathrm{Se}_{56}$ идентифицированы два зарядовых состояния примесных атомов железа $-{ }^{57} \mathrm{Fe}^{3+}$ и ${ }^{57} \mathrm{Fe}^{2+}$. При этом отмечено отсутствие электронного обмена между центрами $\mathrm{Fe}^{2+}$ и $\mathrm{Fe}^{3+}$. В щели подвижности стекол примесные атомы железа образуют одноэлектронные донорные уровни: ионы $\mathrm{Fe}^{2+}$ отвечают нейтральным, а ионы $\mathrm{Fe}^{3+}-$ ионизованным центрам. Электроны донорных уровней железа заполняют локальные состояния в щели подвижности, лежащие выше уровня Ферми, так что происходит сдвиг уровня Ферми от середины щели подвижности (в чистом стекле) к уровню железа (в легированном стекле).

При закалке расплава $\mathrm{Ge}_{28.5} \mathrm{~Pb}_{13.0} \mathrm{Fe}_{2.0} \mathrm{~S}_{56.5}$ (при выливании на металлическую плиту) доля $\mathrm{Fe}^{2+}$ в мессбауэровском спектре возрастает, свидетельствуя в пользу того, что стекло представляет собой микронеоднородный твердый раствор с проводимостью, контролируемой мелкодисперсной железосодержащей фазой, являющейся узкозонным полупроводником [18].

Можно предположить, что при меньших концентрациях железа его атомы встраиваются в сетку стекла за счет взаимодействия неподеленных электронных пар халькогена и $d$-орбиталей переходного металла. В таком случае перенос заряда может быть связан с обменом электронами не только между атомами германия, но и между атомами германия и железа, также пребывающего в двух зарядовых состояниях. Значит, протекание процессов переноса заряда тоже будет определяться характером встраивания атомов железа в сетку стекла, т.е. важную роль будет играть концентрация НЭП. Представляет интерес выяснить, при каких предельных концентрациях примеси железа можно будет обеспечивать существование однофазной системы, кинетические свойства которой определяются взаимодействием НЭП халькогена (серы) с атомами Fe.

Как известно, долю НЭП в халькогенидной системе можно определить соотношением [19]

$$
L=V-m,
$$

где $V$ - число валентных единиц в системе, $m-$ среднее координационное число, определяемое по значениям координационных чисел элементов, входящих в систему, $N_{\mathrm{Ge}}, N_{\mathrm{Pb}}, N_{\mathrm{S}}, N_{\mathrm{Fe}}$, как

$$
m=\frac{a N_{\mathrm{Ge}}+b N_{\mathrm{Pb}}+c N_{\mathrm{S}}+d N_{\mathrm{Fe}}}{a+b+c+d} .
$$

Согласно критерию Дембовского [20], способность к стеклообразованию определяется долей НЭП, а именно в хороших стеклообразователях доля неподеленных пар от полного числа валентных электронов от полного числа валентных электронов $\Phi=($ ВЭК-К)/ВЭК (где ВЭК - полное чисто валентных электронов, К - координационное число) должна быть не ниже 0.50-0.66. При этом отмечалось, что приведенная величина определена не однозначно, так как, во-первых, координационные числа в кристалле и стекле того же состава могут различаться и, во-вторых, в стекле может быть трудно однозначно определить первую координационную сферу. Впоследствии в работе [21] было обращено внимание на то, что способность к стеклообразованию зависит также от сложности состава; в частности было показано, что для бинарных систем доля НЭП должна быть > 2.6 и вместе с тем для тройных соединений достаточно, чтобы их число было $>1$. Для исследуемого состава $\mathrm{Ge}_{28.5} \mathrm{~Pb}_{14.5} \mathrm{Fe}_{0.5} \mathrm{~S}_{56.5}$ получаем значение $L=1.14$, что говорит о хорошей стеклообразующей способности исследуемой системы $\mathrm{Ge}_{28.5} \mathrm{~Pb}_{15} \mathrm{~S}_{56.5}$ с примесью железа. Следует также иметь в виду, что для тонких пленок условия получения аморфной фазы являются менее жесткими, чем для объемного материала. Так, путем магнетронного распыления легко получаются аморфные пленки GeTe, в то время как получить аморфный GeTe в объемной фазе не представляется возможным [22].

\section{5. Заключение}

Обнаруженная степенная зависимость удельной проводимости от частоты и уменьшение показателя степени $s$ с ростом температуры позволяют предположить сушествование прыжкового механизма проводимости, согласно модели СВН (электроны совершают прыжки между локализованными состояниями, преодолевая потенциальный барьер). Перенос заряда в исследуемой гибридной системе $\mathrm{Ge}_{28.5} \mathrm{~Pb}_{14.5} \mathrm{Fe}_{0.5} \mathrm{~S}_{56.5}$ является 
термически активированным процессом, температурная зависимость удельной проводимости для разных частот подчиняется закону Аррениуса с энергией активации $E_{a}=(0.64 \pm 0.02)$ эВ.

\section{Финансирование работы}

Исследование выполнено при финансовой поддержке РФФИ в рамках научного проекта № 19-53-26017 (Чехия_а) и Чешского научного фонда (20-23392J).

\section{Конфликт интересов}

Авторы заявляют, что у них нет конфликта интересов.

\section{Список литературы}

[1] D. Cha, H. Kim, Y. Hwang, J. Jeong, J. Kim. Appl. Optics, 51 (23), 5649 (2012).

[2] G.E. Snopatin., V.S. Shiryaev, V.G. Plotnichenko, E.M. Dianov, M.F. Churbanov. Inorg. Mater., 45 (13), 1439 (2009).

[3] J. Charrier, M.L. Brandily, H. Lhermite, K. Michel, B. Bureau, F. Verger, V. Nazabal. Sensors Actuators B, 173, 468 (2012).

[4] B. Zhang, W. Guo, Y. Yu, C. Zhai, S. Qi, A. Yang, L. Li, Z. Yang, R. Wang, D. Tang, G. Tao, B. Luther-Davies. J. Am. Ceram. Soc., 98 (5), 1389 (2015).

[5] N.F. Mott. Contemp. Phys., 18 (3), 225 (1977).

[6] M. Kastner. Phys. Rev. Lett., 28 (6), 355 (1972).

[7] A.V. Kolobov, H. Oyanagi, K. Tanaka, K. Tanaka. Phys. Rev. B, 55 (2), 726 (1997).

[8] S.R. Ovshinsky. In: Insulating and Semiconducting Glasses, ed. by P. Boolchand (Wold Scientific, 2000) p. 729.

[9] A.V. Kolobov, P. Fons, J. Tominaga, S.R. Ovshinsky. Phys. Rev. B, 87 (16), 165206 (2013).

[10] J.E. Moore. Nature, 464 (7286), 194 (2010).

[11] A.K. Geim, I.V. Grigorieva. Nature, 499 (7459), 419 (2013).

[12] E. Gibney. Nature, 522 (7556), 274 (2015).

[13] Н. Мотт, Э. Дэвис. Электронные прощессы в некристаллических веществах (М., Мир, 1982) т. 1, с. 368.

[14] S.R. Elliot. Adv. Phys., 36 (2), 135 (1987).

[15] I.G. Austin, N.F. Mott. Adv. Phys., 18 (71), 41 (1969).

[16] M.A. Afifi, N.A. Hegab, A.E. Bekheat. Vacuum, 46 (4), 335 (1995).

[17] Г.А. Бордовский, В.А. Извозчиков. Естественно-неупорядоченный полупроводниковый кристалл (СПб., Образование, 1997) с. 422.

[18] G.A. Bordovskii, R.A. Castro. Glass Phys. Chem., 32 (3), 315 (2006).

[19] P. Sharma, S.C. Katyal. Phys. B (Amsterdam, Neth.), 403 (19-20), 3667 (2008).

[20] С.А. Дембовский, Е.А. Чечеткина. Стеклообразование (М., Наука, 1990) с. 279.

[21] L. Zhenhua. J. Non-Cryst. Sol., 127 (3), 298 (1991).

[22] A.V. Kolobov, J. Tominaga. Chalcogenides: metastability and phase-change (Springer, 2012) p. 181.

\section{Charge transfer in thin layers of glassy $\mathbf{G e}_{28.5} \mathbf{P b}_{\mathbf{1 4 . 5}} \mathbf{F e}_{\mathbf{0 . 5}} \mathbf{S}_{\mathbf{5 6 . 5}}$}

R.A. Castro-Arata ${ }^{1}$, G.I. Grabko ${ }^{2}$, A.A. Kononov ${ }^{1}$, N.I. Anisimova ${ }^{1}$, M. Krbal ${ }^{3}$, A.V. Kolobov ${ }^{1,4}$

${ }^{1}$ Herzen State Pedagogical University of Russia, 191186 St. Petersburg, Russia

2 Transbaikal State University, 672039 Chita, Russia

3 University of Pardubice, 53002 Pardubice, Czech Republic

${ }^{4}$ National Institute of Advanced Industrial Science \& Technology, 305-8565 Tsukuba, Japan

Abstract The results of investigation of charge transfer processes in thin layers of vitreous $\mathrm{Ge}_{28.5} \mathrm{~Pb}_{14.5} \mathrm{Fe}_{0.5} \mathrm{~S}_{56.5}$ are presented. The power-law dependence of specific conductivity on frequency and a decrease of the power exponent $s$ value with increasing temperature were found. The charge transfer is a thermally activated process with the activation energy $E_{a}=(0.64 \pm 0.02) \mathrm{eV}$. The results obtained are explained within the correlated barrier hopping model $(\mathrm{CBH})$ of conductivity in disordered systems. The calculated concentration of lone-pair electrons $L$ demonstrates that the material remains in the glass-forming region. 